\title{
Prostate Cancer Specific Exosomal miRNAs in Saliva: a Way to a New and Dependable Screening Method
}

\section{Christoph Lüdemann ( $\square$ Christoph.luedemann@uni-wh.de )}

University of Witten/Herdecke: Universitat Witten/Herdecke

Jan-Ludwig Reinersmann

University of Witten/Herdecke: Universitat Witten/Herdecke

Claudia Klinger

University of Witten/Herdecke: Universitat Witten/Herdecke

\section{Stephan Degener}

HELIOS Hospital Wuppertal: HELIOS Universitatsklinikum Wuppertal

\section{Nici Marcus Dreger}

HELIOS Hospital Wuppertal: HELIOS Universitatsklinikum Wuppertal

\section{Stephan Roth}

HELIOS Hospital Wuppertal: HELIOS Universitatsklinikum Wuppertal

\section{Michael Kaufmann}

University of Witten/Herdecke: Universitat Witten/Herdecke

\section{Andreas Savelsbergh}

University of Witten/Herdecke: Universitat Witten/Herdecke

\section{Research}

Keywords: Salivary exosomes, miRNA, biomarkers, prostate cancer, hsa-mir-331, hsa-mir-200b

Posted Date: December 9th, 2020

DOI: https://doi.org/10.21203/rs.3.rs-122436/v1

License: (1) (1) This work is licensed under a Creative Commons Attribution 4.0 International License. Read Full License 


\section{Abstract}

Background: Today, prostate cancer (PCa) is by far the most common cancer type among men. Diagnostic tests for PCa usually involve blood level testing of prostate-specific antigen (PSA) and transrectal, ultrasound-guided biopsy of the prostatic gland. Both diagnostic tools are unsatisfying in terms of low specificity and poor sensitivity. A general characterization of prostate cancer is difficult since the course of the disease varies individually from latent slow growing to aggressive and rapidly lethal tumors. However, no matter the severity of the disease, a possibility to increase the prognostic chances is the early detection. Regular cancer screening can be an appropriate concept if the tools are right. So far, PSA reaches its limits when it comes to an accurate distinction between cancerous glands and benign processes. miRNAs offer hope to overcome these drawbacks by virtue of their cancer-specific expression. Circulating miRNAs are an active area of current investigation and hold promise to serve a wide range of clinical applications and unwrap a new era in cancer diagnosis and therapeutics. miRNAs have proven their diagnostic potential of becoming a biomolecule of clinical relevance in a variety of human body fluid like blood/serum, urine, and saliva.

Methods: We measured expression levels of 16 circulating prostate cancer specific miRNAs in saliva exosomes via qRT-PCR and compared differences between men suffering from prostate cancer and men suffering from other diseases of the urogenital tract, which formed the no-cancer control group. Differentiation strength of significant sequences was determined via ROC curve analysis.

Results: hsa-mir-331 and hsa-mir-200b were significantly reduced in patients suffering from prostate cancer compared to the no-caner control group. Diagnostic quality of determined cut off values showed moderate differentiation strength with dependable diagnostic properties. The other 14 examined prostate cancer specific microRNAs showed no significant group differences.

Conclusions: hsa-mir-331 and hsa-mir-200b are promising biomarkers for a reliable non-invasive and saliva-based test method in prostate cancer diagnostics. In a synopsis of the results in other studies and against the background of the chastening results of the other miRNAs analyzed, this is exciting news.

\section{Background}

Today, prostate cancer (PCa) is by far the most common cancer type among men. Diagnostic tests for PCa usually involve blood level testing of prostate-specific antigen (PSA) and transrectal, ultrasoundguided biopsy of the prostatic gland. Both diagnostic tools are unsatisfying in terms of low specificity and poor sensitivity [1]. A general characterization of prostate cancer is difficult since the course of the disease varies individually from latent slow growing to aggressive and rapidly lethal tumors. However, no matter the severity of the disease, a possibility to increase the prognostic chances is the early detection. Regular cancer screening can be an appropriate concept if the tools are right [2]. So far, PSA reaches its limits when it comes to an accurate distinction between cancerous glands and benign processes. miRNAs offer hope to overcome these drawbacks by virtue of their cancer-specific expression and high 
stability. Circulating miRNAs are an active area of current investigation and hold promise to serve a wide range of clinical applications and unwrap a new era in cancer diagnosis and therapeutics [3]. miRNAs have proven their diagnostic potential of becoming a biomolecule of clinical relevance in a variety of human body fluid like blood/serum, urine, and saliva [4] [5].

\section{Methods}

\section{Literature review}

Literature research based on articles published up to January 2014, according to the Preferred Reporting Items for Systematic Review and Meta-analysis statement [7]. Publications featuring prostate cancer specific aberration of microRNA expression were further selected focusing on results obtained from body fluid examination and measurements. We identified 16 microRNAs with promising diagnostic relevance

for further examination in saliva samples based on the following four publications, reporting either up- or downregulation of different miRNAs in body fluids (e.g., urine, blood, plasma) of prostate cancer patients. Bryant et al. (2012), Brase et al. (2011), Chen et al. (2012) and Moltzahn et al. (2011) ([8], [9], [10],[11]). Table 1 presents each source of literature and miRNAs withdrawn from these sources.

\section{Research subjects}

Patients were continually recruited from the urology department of the Helios University Hospital Wuppertal from spring 2013 until summer 2017. Included were men of any age with elevated PSA-blood serum levels suspected to suffer from prostate cancer admitted for result clarification via prostate biopsy. Exclusion criteria were secondary diagnosis, e.g., infectious diseases (HIV, hepatitis, tuberculosis etc.), Sicca Syndrome, prior interventions like operations of the salivary gland or prostate, urinary diversion with intestine tissue, radiotherapy of the pelvic region or antiandrogen therapy as well as a patient's nonconsent, since the participation in the study was voluntary.

The sample size of the cohort was planned to be 100 patients with equal distribution between diagnosed prostate-cancer patients and no-cancer patients. Patients proving cancer free via prostate stance biopsy were then added to the no-cancer control group. Origin of their PSA level elevation were different medical issue or diseases, such as prostatitis, urinary occlusion or secondary genesis, but most commonly benign prostate hyperplasia (BPH) [12]. In all cases, patient history was documented, routine blood and urine samples were taken, a routine urine bacterial culture was acquired and two to three days before prostate biopsy preventive antibiotic therapy (Ciprofloxacin) was given. Each patient gave an additional $5 \mathrm{ml}$ whole saliva sample. Patients were instructed to refrain for two hours from smoking, drinking or eating to keep all samples pure, genuine and undiluted. In total, the study involved 86 patients. 50 patients were later diagnosed with histologically validated prostate cancer. 36 patients were validated histologically as well but missed the criteria for prostate cancer, presenting other causes for elevated PSA levels like BPH. Only 74 patients were able to provide whole saliva samples valid for further expression level analysis. This cohort presents the study sample for analysis of concentration differences of 16 miRNAs between cancer and no-cancer patients. 


\section{Isolation of exosomes and extraction of miRNA}

The extraction of miRNA from salivary exosomes was based on the model of Gallo et al. (3), with modifications as described by Wiegand et al [13]. Immediately after collection, the saliva samples were frozen at $-20^{\circ} \mathrm{C}$. For analysis, the samples were thawed at $0{ }^{\circ} \mathrm{C}$ and centrifuged at $1.000 \times \mathrm{G}$ for 2 minutes to remove food and cell debris. Two milliliters of the supernatant were centrifuged at $1.500 \mathrm{rpm}$ for 10 minutes (Mini-Spin), additionally for saliva with high viscosity it was followed by a centrifugation at $4.700 \mathrm{rpm}$ for 10 minutes (Mini-Spin). The supernatant was then transferred into a new tube and centrifuged at $13.500 \mathrm{rpm}$ for 15 minutes (Mini-Spin) at first and then at $16.000 \mathrm{x} \mathrm{G}$ for 60 minutes at $4{ }^{\circ} \mathrm{C}$ in a SS55S Rotor in an MGX-120 Ultracentrifuge (Sorvall). The supernatant was then discarded. One milliliter of Trizol reagent was added to the pellet \& mixed. Two hundred microliters of chloroform were added to the Trizol and vortexed for 30 seconds, before the mixture was allowed to rest for 3 minutes and subsequently centrifuged at $13.500 \mathrm{rpm}$ for 15 minutes (Mini-Spin). The upper, clear phase was transferred into a new Eppendorf tube and $500 \mu \mathrm{l}$ of Isopropanol was added. The mixture was then incubated at $-20^{\circ} \mathrm{C}$ for 20 minutes. After that, the mixture was centrifuged at $13.500 \mathrm{rpm}$ for 15 minutes (Mini-Spin) and the supernatant was again discarded. The pellet was washed with $1 \mathrm{ml}$ of $75 \%$ ethanol, followed by a centrifugation at $11.000 \mathrm{rpm}$ for 5 minutes (Mini-Spin) and discarding of the supernatant. The pellet containing the miRNA was dried for 20 minutes with the cover lid opened at room temperature. Twenty-five microliters of $\mathrm{H}_{2} \mathrm{O}$ (RNAse free) were added to the pellet followed by vortexing. At last, the mixture was incubated at $60^{\circ} \mathrm{C}$ for 15 minutes, followed by a brief centrifugation due to condensed water at the cover lid.

\section{qRT-PCR}

Real time quantitative PCR was used to detect and quantify miRNAs of interest. For the RT reaction, the miScript II RT Kit (50) from Qiagen ${ }^{\circledR}$ was used. In total, $8 \mu$ l Master Mix were combined with $12 \mu \mathrm{l}$ template RNA, adding up to $20 \mu \mathrm{l}$ RT-mix for the reverse transcriptase reaction protocol.

For the qPCR, the miScript SYBR Green PCR Kit from Qiagen ${ }^{\circledR}$ and the primer assays MIR375, MIR484, MIR2110, MIR130B, MIR301A, MIR326, MIR106A, MIR331, MIR107, MIR141, MIR432, MIR574, MIR625, MIR181, MIR200b, MIR622 were used. The standardizing assay was for RNU6-6P RNA. The qPCR preparation included $145 \mu \mathrm{l}$ of SYBR Green, $29 \mu \mathrm{l}$ of U-primer, $29 \mu \mathrm{l}$ of $\mathrm{H}_{2} \mathrm{O}$ (RNAse free) and $29 \mu \mathrm{l}$ of RTdilution. Each qPCR assay consisted of $8 \mu \mathrm{l}$ master mix and $2 \mu \mathrm{l}$ miRNA-specific Primer or control primer. qPCR cycling conditions were used as in the miScript SYBR Green PCR Kit handbook for the detection of mature miRNA. The data from qRT-PCR was documented and processed with qPCRSoft 21 program. From each PCR, melting curve and melting temperature were analyzed to verify specificity and quality of the reaction. In case of sample contamination or dysfunction of reaction components (enzymes, nucleotides, etc.) melting curves showed aberrant melting temperature or curve outline and qRT-PCR was redone. If more than four different microRNA sequences from one sample showed no valid data (no signal detectable or invalid melting curve), the whole sample was discarded. In this case, microRNA extraction from the remaining saliva sample was redone and qRT-PCR performed again. If no valid data 
could be acquired after three trials, the saliva sample was classified as contaminated and the patient was not considered for further microRNA expression analysis.

Independently from that, if the U6 snRNA (control sequence) showed no detectable reaction or an invalid melting curve, the whole data set was discarded and redone.

Valid qRT-PCR data sets provided CT values for further microRNA expression analysis. CT values were condensed using the comparative CT method (2- $\Delta \Delta \mathrm{CT}$-method) [14].

\section{Statistical analysis}

Main focus of the statistical analysis were concentration differences of 16 different microRNA sequences in saliva exosomes between two groups, patients with histologically verified prostate cancer diagnose (cancer group) and patients with no proof of cancer in prostate biopsy (comparison group). Additional documented clinical parameter (age, PSA serum concentration etc.) were checked for significant group differences, too. Statistical analysis was performed using parametric or non-parametric test depending on distribution character of the data. Unless stated otherwise, statistical analyses were conducted in SPSS version 25 (IBM, Armonk, USA). P values $<.05$ were considered significant.

\section{Results}

The statistical analysis of established screening parameters showed no significant difference between patients suffering from prostate cancer and the no-cancer control group. Except for the prostate volume measured via transrectal ultrasound, where Mann-Whitney-U test proves significantly smaller prostate volume of cancer group (mean volume $42.82 \mathrm{ml}, \mathrm{SD} \pm 22.93 \mathrm{ml}$ ) compared to the comparison group (mean volume $74.39 \mathrm{ml}, \mathrm{SD} \pm 47.55 \mathrm{ml})(\mathrm{U}[36,50]=-3.858, \mathrm{p}=.001$ and $\mathrm{t}(84)=-4.087, \mathrm{p}=.001)$. (See table 2)

All 16 microRNA sequences analyzed in this study were detectable in salivary exosomes in both patient groups, therefore part of the comparative analysis. However, 14 out of 16 miRNAs showed no significant difference in expression levels between both groups. hsa-mir-200b and hsa-mir-331 showed statistically significant concentration differences in saliva exosomes between the cancer patients and the no-cancer control group (see table 3). (Mann-Whitney-U-Test: hsa-mir-200b: cancer group mean $\Delta C T=-3.26, S D \pm$ 3.11 and comparison group mean $\Delta C T=-5.11, S D \pm 3.23, U[31,43]=-2,383, p=.017$; hsa-mir-331: cancer group mean $\Delta \mathrm{CT}=-1.78, \mathrm{SD} \pm 2.81$ and comparison group $\Delta \mathrm{CT}=-3.17, \mathrm{SD} \pm 2.84, \mathrm{U} 331,43]=-2.158, \mathrm{p}$ $=.031$ ) [15]. hsa-mir-200b was 3.6-fold reduced and hsa-mir-331 was 2.64-fold reduced in cancer patients compared to patients suffering from other diseases of the urogenital tract. Table 3 shows the summarized mean $\triangle \mathrm{CT}$ values and foldchanges for each microRNA within the cancer group and the comparison group respectively.

Figure 1 presents boxplots of hsa-mir-200b and hsa-mir-331 with significant expression level differences between the comparison group and the cancer group. 
We performed ROC curve analysis to validate diagnostic accuracy for both markers and determined an area under the curve of .663 for hsa-mir-200b and .648 for hsa-mir-331 [16]. Further examination of the data based on the Youden-Index method determined a cut-off value of $\Delta C T=-5.5$ for hsa-mir-200b with a sensitivity of $81 \%$ and specificity of $55 \%$ and a cut-off value of $\Delta \mathrm{CT}=-2.8$ for hsa-mir-331 with a sensitivity of $74 \%$ and specificity of $58 \%$ [17].

Using absolute case numbers of our study patients, we calculated prositive and negative predictive values for each microRNA biomarkers (see table 4) [18]. hsa-mir-200b showed a positive predictive value of $71 \%$ to indicate prostate cancer if CT-value was bigger than - 5.5. miRNA hsa-mir-331 showed a positive predictive value as well of $71 \%$ for prostate cancer when CT-value was detected over -2.8 . In 39 cases further cross table analysis of case numbers and microRNA test results revealed patients that showed positive test results for both microRNA sequences (hsa-mir-200b + AND hsa-mir-331+). Thus, meaning a measured CT value above the calculated cut off score. Only 15 patients tested positive for only one of both sequences, either hsa-mir-200b or hsa-mir-331. When it comes to diagnostic accuracy, a double positive test result (200b+/331 + OR 200b-/331-) shows similar sensitivity and specificity for prostate cancer to a positive test result of each single sequence $(200 \mathrm{~b}+/ 331$ - or $200 \mathrm{~b}-/ 331+)$.

\section{Discussion}

In this study we aimed to find a new approach to non-invasive prostate cancer screening methods as PSA serum measurements produce high numbers of false positive prostate cancer suspects [19]. We hypothesized that PCa-specific miRNAs measured in saliva exosomes show significant expression level differences between men suffering from prostate cancer and a non-cancer control group enabling a reliable differentiation of both groups in the common preventive screening program. The control group in the present study consisted of men suffering from other diseases of the urogenital tract associated with PSA level elevations. The acquired results confirmed our hypothesis, two out of sixteen examined microRNA sequences (hsa-mir-331, hsa-mir-200b) were significantly reduced in saliva samples from prostate cancer patients compared to the no-cancer control group. These differences correspond with former studies reporting a significant reduction of hsa-mir-200b and hsa-mir-331 in prostate tumor cells compared to healthy prostate tissue samples [20] [21] [22] [23]. Further determination of diagnostic value of both sequences via ROC curve analysis revealed moderate but reliable differentiation strength (AUC of .663 for hsa-mir-200b and .648 for hsa-mir-331) [16]. A recent study from 2017 of Souza et al found similar diagnostic values for hsa-mir-200b with a sensitivity of $67 \%$ and specificity of $75 \%$ for PCa patients, although examining concentration differences in blood serum samples rather than saliva exosomes [24]. A more clinical and comprehensible assessment of the diagnostic strength is the prognostic value of a test method. Both, hsa-mir-200b and hsa-mir-331 achieved a positive predictive value for prostate cancer of $71 \%$, meaning the saliva test results were consistent with the histologic findings from prostate stance biopsy in $70 \%$ of the cases, malign as well as benign diagnose. To our knowledge, this is the first time hsa-mir-200b and hsa-mir-331 were measured in saliva exosomes with the ability to differentiate between men suffering from prostate cancer and a control group. 
Lastly, analysis of case numbers and microRNA test results revealed that most of the patients tested positive not only for one but both sequences (i.e., measured expression level indicating prostate cancer according to cut off value), only 15 patients showed a positive test for only one microRNA, either hsa-mir$200 \mathrm{~b}$ or hsa-mir-331. Significant results for more than one PCa-specific microRNA could indicate the existence of a diagnostic pattern of different microRNA sequences possibly with higher prognostic value for prostate cancer than the diagnostic ability of any microRNA sequence alone. To investigate this hypothesis, we compared sensitivity and specificity of a double positive test result to the diagnostic accuracy of hsa-mir-200b or hsa-mir331 calculated separately and individually. None of those interpretation possibilities showed superiority in discrimination between both groups as sensitivity and specificity were approximately the same. Therefore, we conclude that a microRNA saliva test with a single PCa-specific sequence with high diagnostic value could be more efficient than a combined examination of different microRNAs and development of a diagnostic pattern or panel. Anyhow, for a profound and certain answer to this question, further research on PCa-specific microRNA measured in saliva exosomes is needed.

Although the present study produced promising results indicating a useful augmentation of the standard PCa screening by saliva-based microRNA examination, the overall representative strength of our findings is limited by the composition of the study cohort. The study design included only men classified as prostate cancer suspects due to elevated PSA serum levels admitted to hospital for further verification of a potential cancer threat. Half of the patients were diagnosed with prostate cancer, the others suffered from other diseases of the urogenital tract also accompanied by PSA level elevation. Healthy individuals were not included. Therefore, detected significant group differences do not qualify to assess the identification ability of prostate cancer patients among healthy individuals, which is the purpose of preventive screening programs.

Nevertheless, the study design highlights a problematic aspect of the current PCa screening program to which salivary microRNA tests could provide a solution; that is high numbers of false positive prostate cancer suspects due to low predictive value of PSA serum measurements [25]. Examining the differences between true prostate cancer patients and those falsely suspected to suffer from prostate cancer (control group) after preventive PCa screening, this study revealed a reliable differentiation between both groups via non-invasive measurement of hsa-mir-331 and/or hsa-mir-200b in saliva samples. The current study proved the differentiation ability of those two microRNA sequences via ROC curve analysis and determined cut-off scores with good prognostic value. Augmentation of a conspicuous PSA test result by this method could verify or refute an uncertain cancer suspicion. In consequence, hsa-mir-331 and hsamir-200b concentration measurement in saliva samples could reduce numbers of false positive cancer suspects in the preventive screening program and thereby reduce numbers of falsely indicated prostate biopsies. All in all, two out of the 16 examined microRNAs showed prostate cancer specific changes in expression levels when measured in salivary exosomes. Hsa-mir-200b is a member of the mir-200-family whose involvement in carcinogenesis and aberrant cell development is documented for many different cancer types. Gene targets of hsa-mir-200b in prostate cancer development in particular are among others AMACR, BCL2, GOLM1, OR51E2, SIM2 or Bmi-1. Bmi-1 for example is accountable for abnormal 
cell proliferation, migration and chemosensitivity and is described to be suppressed by hsa-mir-200b [26]. Therefore, loss of or reduction of hsa-mir-200b as measured in PCa patients can induce abnormal cell proliferation in prostate gland tissue. Other research shows, downregulation of hsa-mir-200b can also induce epithelial-mesenchymal transition (EMT) in prostate gland cells. EMT describes the pathological change of epithelial cells from a cobblestone-like, tissue-adherend phenotype to a spindle-like, loosely attached cell character often found in embryonic mesenchymal stem cells leading to more cell motility, invasion of other tissue layers and eventually metastasis to other regions of the body [27] [28]. Recent studies stated, that hsa-mir-200b supports E-cadherin expression by suppressing its transcription repressor ZEB1. Transcriptions factor ZEB1 binds to ZEB-type E-boxes (CACCTG) within E-cadherins promoter region inducing chromatin condensation and gene silencing [29]. E-cadherin is one of the most important members of cell connection molecules like desmosomes or tight junctions and therefore very important to EMT.

A stunning number of studies already revealed important roles of hsa-mir-331 in several cancer types like leukemia, lung, gastric and liver cancer. Some references deal with the role in prostate cancer development and interestingly all of them have suggested a tumor suppressor role for hsa-mir-331 in prostate cancer, unanimously. Shee et al. provided the most recent review of the pathological and physiological roles of the miRNA-331 family in cancer [30]. The findings of Epis et al. (2009) suggest a role for hsa-mir-331 in the development and progression of prostate cancer while focusing on ERBB-2 as a target of this miRNA. They found hsa-mir-331 expression to be decreased in ERBB-2 overexpressing PCa tissue relative to normal adjacent tissue. Furthermore, they have shown that hsa-mir-331 blocked androgen receptor (AR) signaling in PCa cells. A loss of hsa-mir-331 expression could promote the increased ERBB-2 expression and signaling seen in many prostate cancers [21].

Wang et al. (2009) confirmed hsa-mir-331 to play an important role when identifying candidate genes involved in causal pathways of aggressive prostate cancer. They found hsa-mir-331 to be differentially expressed in prostate cancer cell lines and implicated an important part that hsa-mir-331 plays in cell cycle regulation [23].

In a later study, Epis et al. (2011) confirmed the reduced expression of hsa-mir-331 in aggressive prostate cancer. They showed that the RNA-binding protein HuR induces the ERBB-2 expression in prostate cancer by preventing the degradation of ERBB-2 mRNA by hsa-mir-331 [22]. Epis et al. (2011) were also involved in the findings that showed hsa-mir-331 playing an inhibiting part in the growth of prostate cancer cells. hsa-mir-331 seems to repress the expression of the deoxyhypusine hydroxylase (DOHH). This enzyme is an important player in protein modification [22]. When it comes to the aggressiveness of prostate cancer, which can variate widely, Fredsoe et al. (2019) developed a five-miRNA model for predicting prostate cancer aggressiveness using cell-free urine. hsa-mir-331 plays a decisive role in this 5-miRNA orchestra [31].

In epithelial-mesenchymal transition, another target for hsa-mir-331 seem to be Neuropilin 2 and nucleus accumbens-associated protein 1 . The findings of Fujii et al. (2016) indicate these targets for hsa-mir- 
331[32]. White et al. (2012) confirmed hsa-mir-331 to be a tumor suppressor via its regulation of Kallikrein-related peptidases 4 (KLK4) expression [33].

In this study, hsa-mir-331 was one out of two miRNAs to show significantly different results in cancer patients compared with the healthy control group - has-mir-331 was significantly downregulated. When comparing both groups (cancer and comparison group) hsa-mir-331 is decreased by a 2.64 -fold change.

When tested for significance, the different expression of hsa-mir-331 shows a significant level with a pvalue of 0.031 (with a threshold of $p=0.05$ ).

Decreased levels of hsa-mir-331 concur with former studies that mostly showed decreased levels of hsamir-331 in prostate cancer patients [32].

The 4 studies showing results that indicate a downregulation of hsa-mir-331 were studying cell lines rather than body fluids. Epis et al. $(2009,2011)$ worked with prostate cancer tissue and Wang et al. (2009) focused on cancer cell lines [23] [21] [22]. However, the results oppose the findings of Fujii et al. (2016). Looking closely at the study of Fujii et al. (2016), they found that hsa-mir-331 expression was much higher in prostate cancer cells with higher Gleason scores $(7,8$, and 9) [32]. The patient data of the cancer group of this study shows, that $64 \%$ of the prostate cancer patients had higher Gleason scores of 7 to 10 .

In summary, hsa-mir-331 holds true for the hypothesis to be a potential circulating exosome-derived biomarker in saliva for the detection of prostate cancer.

The other 14 examined circulating microRNA presented no significant group differences between cancer and control group, although being described as PCa-specific in preliminary literature research. Included within this paper were studies identifying potential PCa-microRNA. However, all of them examined expression levels in prostate tissue, cancer cell lines or blood serum samples rather than salivary exosomes. Supplementary research on the influence of saliva filtration processes or active microRNA equipment of salivary exosomes were limited but could provide information on missing disease specific differences of certain circulating microRNA. Therefore, occurrence or absence of PCa-specific microRNA in saliva exosomes need to be part of future investigations. Another reason for inconsistent PCa specificity of the other 14 microRNA may derive from the composition of the control group in this study. In contrast to other studies working with healthy individuals for comparison, the present control collective consisted of patients suffering from other diseases associated with PSA level elevations. Especially diseases of the urogenital tract could be influenced by the same microRNA known for prostate cancer carcinogenesis. Hence, the other 14 examined microRNA might be indicators for general urogenital pathologies rather than only prostate cancer. Future research needs to identify and verify PCa specificity of microRNA in salivary exosomes in comparison to other diseases of the urogenital tract to enable a salivary microRNA test method with high diagnostic accuracy.

\section{Conclusions}


All recruited patients of the present study were suspected to suffer from prostate cancer due to elevated PSA serum levels in preventive screening procedures. In the end, only half of these suspects were actually diagnosed with malign prostate cancer, the others showed elevated PSA levels due to benign reasons of other kind. Nevertheless, a differentiation of both groups on the basis of the standard screening parameters and their assessment by medical experts proved insufficient. Contrary to that, expression level analysis of two different microRNAs measured in exosomes from whole saliva samples revealed not only significant group differences but presented reliable enough to indicate prostate cancer or no-cancer with a prognostic value of $70 \%$.

These findings suggest that exosome derived salivary microRNA could serve as a reliable evaluation tool of prostate cancer screening results (e.g. PSA serum measurements) under the concept of liquid biopsy [34]. This new and non-invasive approach could substantiate or contradict a prostate cancer suspicion and increases overall sensitivity and specificity of prostate cancer screenings. It could advise the clinician and/or the patient in the decision-making process for further diagnostic steps and potentially reduce the number of false positive prostate biopsies.

We believe that further investigation of salivary exosomes and microRNA expression assays might reveal more potential candidates of PCa specific circulating microRNAs enabling a non-invasive, saliva-based test method with high prognostic value to indicate prostate cancer. In this context, hsa-mir-200b and hsamir-331 are the first two promising candidates for a supplementary part in prostate cancer diagnostics.

\section{Abbreviations}

$\mathrm{PCa}$ - prostate cancer

PSA - prostate-specific antigen

qRT-PCR - quantitative real time polymerase chain reaction

ROC - receiver operations characteristic

$\mathrm{BPH}$ - benign prostate hyperplasia

snRNA - small nuclear ribonucleic acid

CT - cycle threshold

AMACR - Alpha-Methylacyl-CoA Racemase

BCL2 - B-cell lymphoma protein 2

GOLM1 - Golgi membrane protein 1

OR51E2 - Olfactory Receptor Family 51 Subfamily E Member 2 
SIM2 - single-minded homolog 2

Bmi-1 - B cell-specific Moloney murine leukemia virus integration site 1

EMT - epithelial mesenchymal transition

AR - androgen receptor

ERBB - receptor tyrosine kinases

DOHH - deoxyhypusine hydroxylase

HuR - human antigen $\mathrm{R}$

KLK4 - Kallikrein-related peptidases 4

\section{Declarations}

\section{Ethics approval and consent to participate}

The study was approved by the ethics committee of the University of Witten/Herdecke by the ethics vote/reference No. $07 / 2014$. The study is subject to the Declaration of Helsinki in its last revised version of 2013.

\section{Consent for publication}

Not applicable

\section{Availability of data and materials}

The datasets used and/or analyzed during the current study are available from the corresponding author on reasonable request.

\section{Competing interests}

The authors declare that they have no competing interests

\section{Funding}

This study received funding from the Helios Forschungsförderung, Berlin, Germany (Grant ID 000030) and the Interne Forschungsförderung, Witten/Herdecke University, Witten, Germany.

\section{Author's contribution}

$\mathrm{CL}$ and JR performed the experimental procedures, data analysis and wrote the manuscript. AS, CK and MK were responsible for the design of the study. CK was responsible for the study protocol and ethic 
approval. AS, CK and MK revised the manuscript. SD, ND and SR participated in the selection of patients and collection of patients saliva. All authors gave final approvement to the manuscript and agree to be accountable for its content.

\section{Acknowledgments}

We thank F. Gholamrezaei and M. Wahlkamp for expert technical assistance.

\section{References}

1. World Health Organization, GLOBOCAN 2012. 2015.

2. Leitlinienprogramm Onkologie (Deutsche Krebsgesellschaft, D.K., AWMF), Interdisziplinäre Leitlinie der Qualität S3 zur Früherkennung, Diagnose und Therapie der verschiedenen Stadien des Prostatakarzinoms. 2018.

3. Fabris, L., et al., The Potential of MicroRNAs as Prostate Cancer Biomarkers. European Journal of Urology, 2016. 70(2): p. 312-322.

4. Kroh, E., et al., Analysis of circulating microRNA biomarkers in plasma and serum using quantitative reverse transcription-PCR (qRT-PCR). Methods, 2010. 50(4): p. 298-301.

5. Sita-Lumsden, A., et al., Circulating microRNAs as potential new biomarkers for prostate cancer. British Journal of Cancer, 2013. 108: p. 1925-1930.

6. Jackson, B., A. Grabowska, and H. Ratan, MicroRNA in prostate cancer: functional importance and potential as circulating biomarkers. Cancer, 2014. 14(930).

7. Moher, D., et al., Preferred reporting items for systematic reviews and meta-analyses: the PRISMA statement. BMJ, 2009. 339.

8. Bryant, R.J., et al., Changes in circulating microRNA levels associated with prostate cancer. British Journal of Cancer, 2012(106): p. 768-774.

9. Brase, J.C., et al., Circulating miRNAs are correlated with tumor progression in prostate cancer. International Journal of Cancer, 2011. 128: p. 608-616.

10. Moltzahn, F., et al., Microfluidic-based multiplex qRT-PCR identifies diagnostic and prognostic microRNA signatures in the sera of prostate cancer patients. Cancer Res, 2011. 71(2): p. 550-60.

11. Chen, C.-Z., et al., A panel of five circulating microRNAs as potential biomarkers for prostate cancer. The Prostate, 2012. 72(13): p. 1443-1452.

12. Nadler, R.B., et al., Effect of Inflammation and Benign Prostatic Hyperplasia on Elevated Serum Prostate Specific Antigen Levels. Journal of Urology, 1995. 154(2): p. 407-413.

13. Wiegand, C., et al., Stress-associated changes in salivary microRNAs can be detected in response to the Trier Social Stress Test: An exploratory study. Sci Rep, 2018. 8: p. 7112.

14. Schmittgen, T.D. and K.J. Livak, Analyzing real-time PCR data by the comparative CT method. Nature Protocols, 2008. 3(6): p. 1101-1108. 
15. Mann, H.B. and D.R. Whitney, On a Test of Whether one of Two Random Variables is Stochastically Larger than the Other. The Annals of Mathematical Statistics, 1947. 18(1): p. 50-60.

16. Zweig, M. and G. Campbell, Receiver-operating characteristic (ROC) plots: a fundamental evaluation tool in clinical medicine. Clin Chem., 1993. 39(4): p. 561-577.

17. Ruopp, M., et al., Youden Index and optimal cut-point estimated from observations affected by a lower limit of detection. Biom J., 2008. 50(3): p. 419-430.

18. Akobeng, A., Understanding diagnostic tests 1: sensitivity, specificity and predictive values. Acta Paediatrica, 2007. 96(3): p. 338-341.

19. Vezyraki, P., et al., Impact of total PSA and percent free PSA in the differentiation of prostate disease: a retrospective comparative study implicating neoplastic and non-neoplastic entities. J BUON, 2019. 24(5): p. 2107-2113.

20. Katz, B., et al., Comprehensive Study of Gene and microRNA Expression Related to EpithelialMesenchymal Transition in Prostate Cancer. PLoS One, 2014. 9(11).

21. Epis, M.R., et al., miR-331-3p regulates ERBB-2 expression and androgen receptor signaling in prostate cancer. J Biol Chem, 2009. 284(37): p. 24696-24704.

22. Epis, M.R., et al., The RNA-binding Protein HuR Opposes the Repression of ERBB-2 Gene Expression by MicroRNA miR-331-3p in Prostate Cancer Cells. The Journal of Biological Chemistry, 2011. 286: p. $41442-41454$.

23. Wang, L., et al., Gene networks and microRNAs implicated in aggressive prostate cancer. Cancer Res, 2009. 69(24): p. 9490-7.

24. Souza, M., et al., Circulating mRNAs and miRNAs as candidate markers for the diagnosis and prognosis of prostate cancer. PLoS One, 2017. 12(9).

25. Pezaro, C., H.H. Woo, and I.D. Davis, Prostate cancer: measuring PSA. Intern Med J, 2014. 44(5): p. 433-440.

26. Yu, J., et al., miR-200b suppresses cell proliferation, migration and enhances chemosensitivity in prostate cancer by regulating Bmi-1. Oncol Rep, 2014. 31(2): p. 910-918.

27. Bracken, C., et al., A Double-Negative Feedback Loop between ZEB1-SIP1 and the microRNA-200 Family Regulates Epithelial-Mesenchymal Transition. Cancer Res, 2008. 68(19): p. 7846-7854.

28. Hugo, H., et al., Epithelial-mesenchymal and mesenchymal-epithelial transitions in carcinoma progression. . J Cell Physiol., 2007. 213(2): p. 374-83.

29. Peinado, H., D. Olmeda, and A. Cano, Snail, Zeb and bHLH factors in tumour progression: an alliance against the epithelial phenotype? Nature Reviews Cancer, 2007. 7: p. 415-428.

30. Shee, S.M.E., et al., The roles of microRNA-331 Family in Cancers. Journal of Cancer Research and Practice, 2019. 6(1): p. 1-6.

31. Fredsoe, J., et al., A five-microRNA model ( $\mathrm{pCaP}$ ) for predicting prostate cancer aggressiveness using cell-free urine. International Journal of Cancer, 2019. 145(9). 
32. Fujii, T., et al., Syndecan-1 up-regulates microRNA-331-3p and mediates epithelial-to-mesenchymal transition in prostate cancer. Mol Carcinog., 2016. 55(9): p. 1378-86.

33. White, N.M.A., et al., The miRNA-kallikrein axis of interaction: a new dimension in the pathogenesis of prostate cancer. Biological Chemistry, 2012. 393(5).

34. Crowley, E., et al., Liquid biopsy: monitoring cancer-genetics in the blood. Nat Rev Clin Oncol, 2013. 10(8): p. 472-84.

\section{Tables}

Due to technical limitations, table PDF is only available as a download in the Supplemental Files section.

\section{Figures}
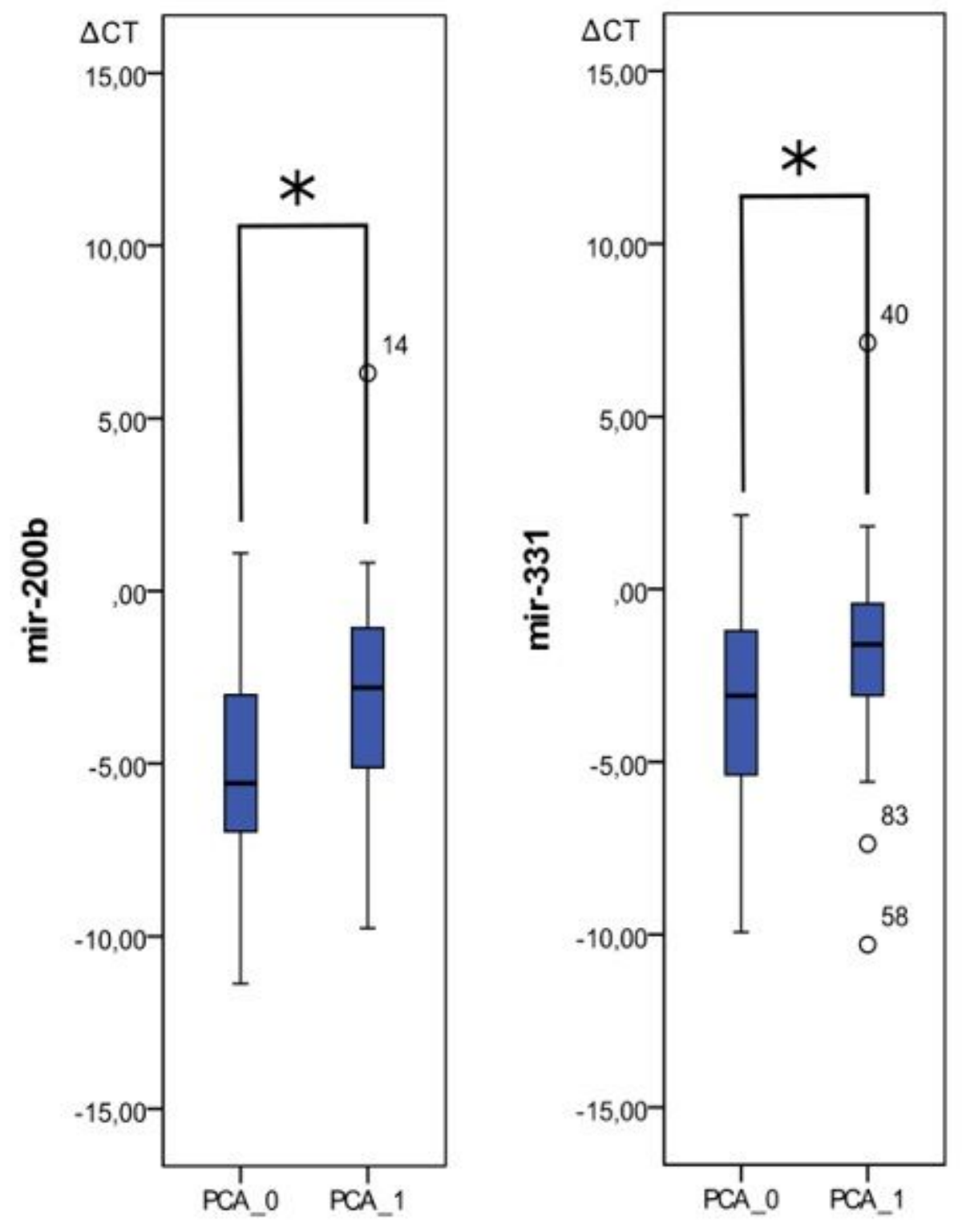
Figure 1

Boxplots of hsa-mir-200b and hsa-mir-331 with significant expression level differences between the comparison group and the cancer group.
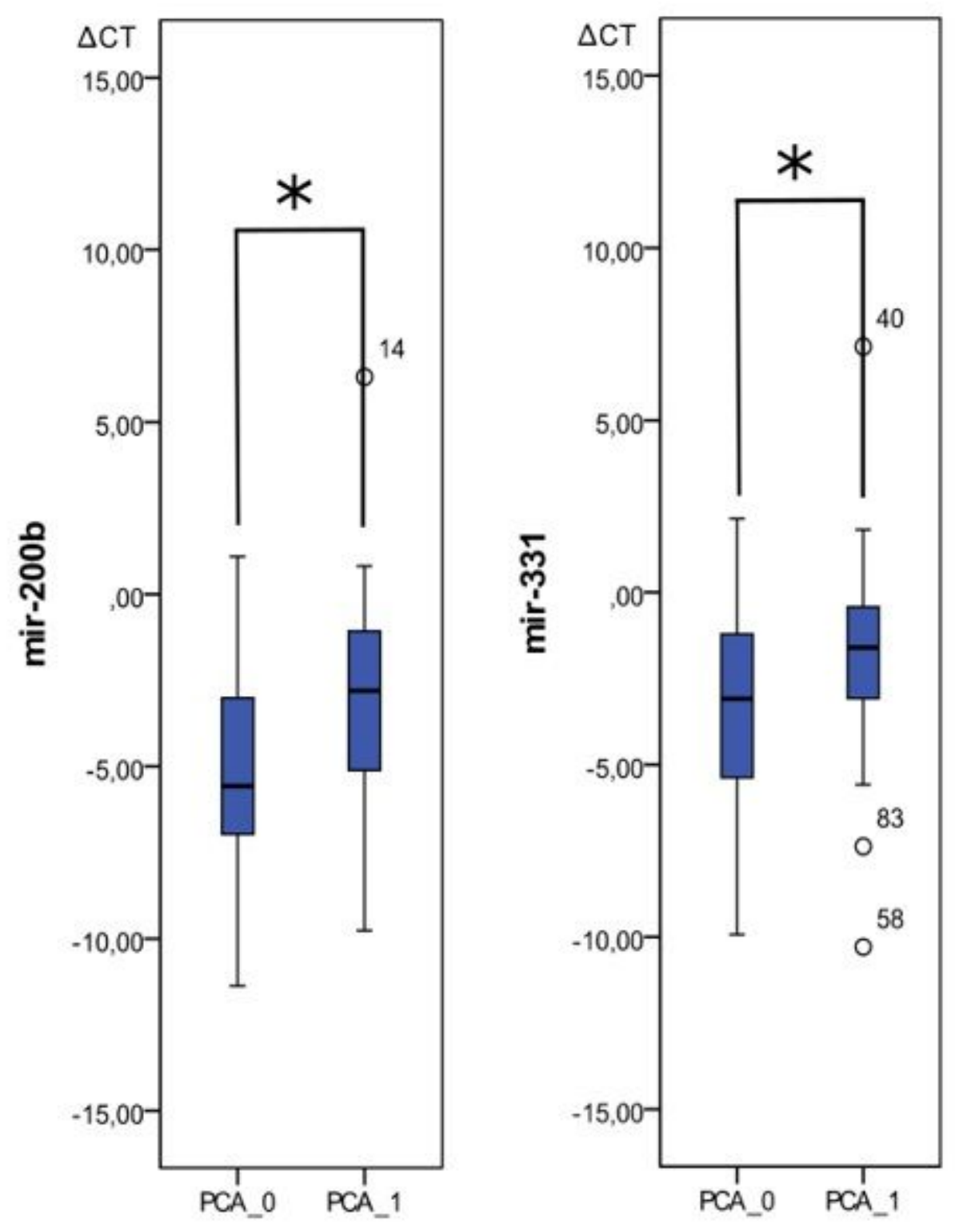

Figure 1

Boxplots of hsa-mir-200b and hsa-mir-331 with significant expression level differences between the comparison group and the cancer group. 
ROC curve mir-200b

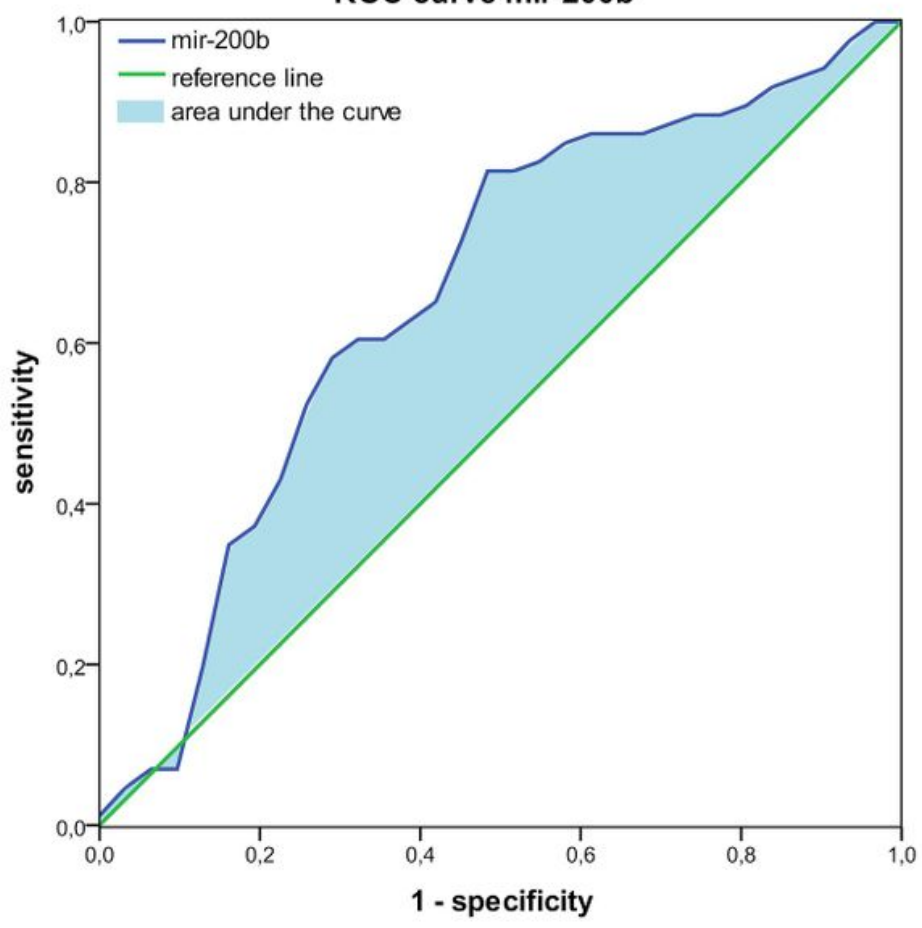

Figure 2

ROC-curves of hsa-mir-200b and hsa-mir-331

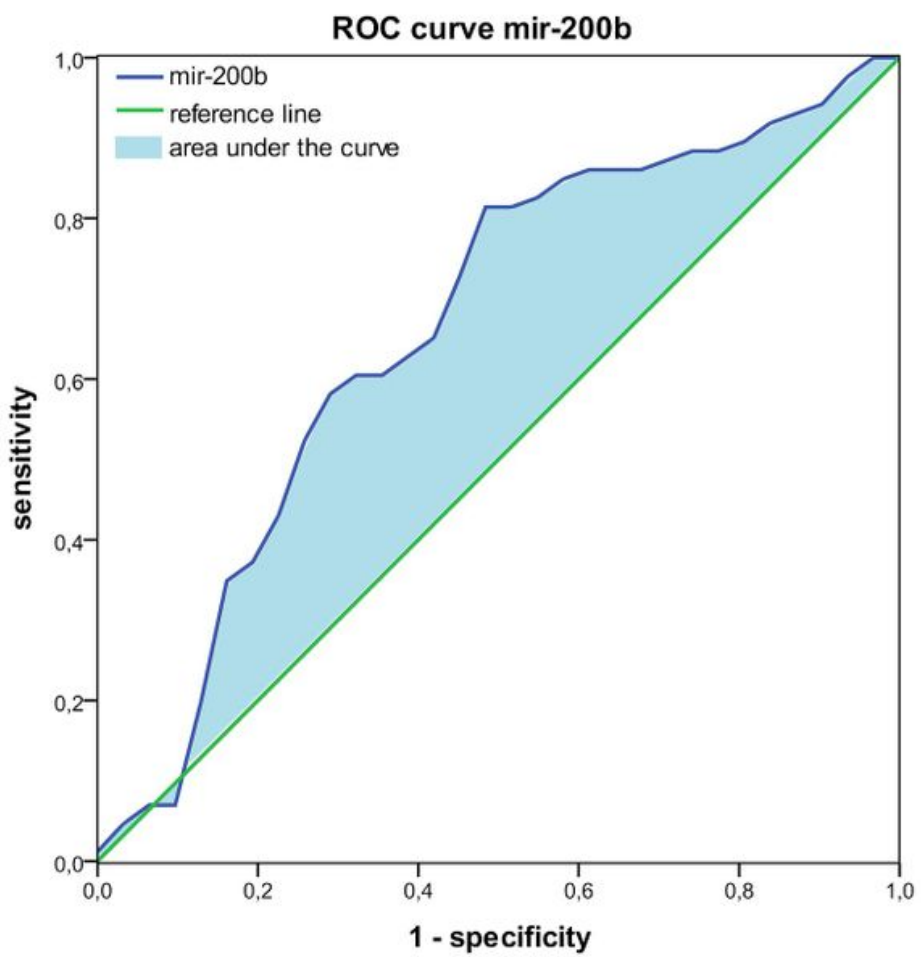

ROC curve mir-331
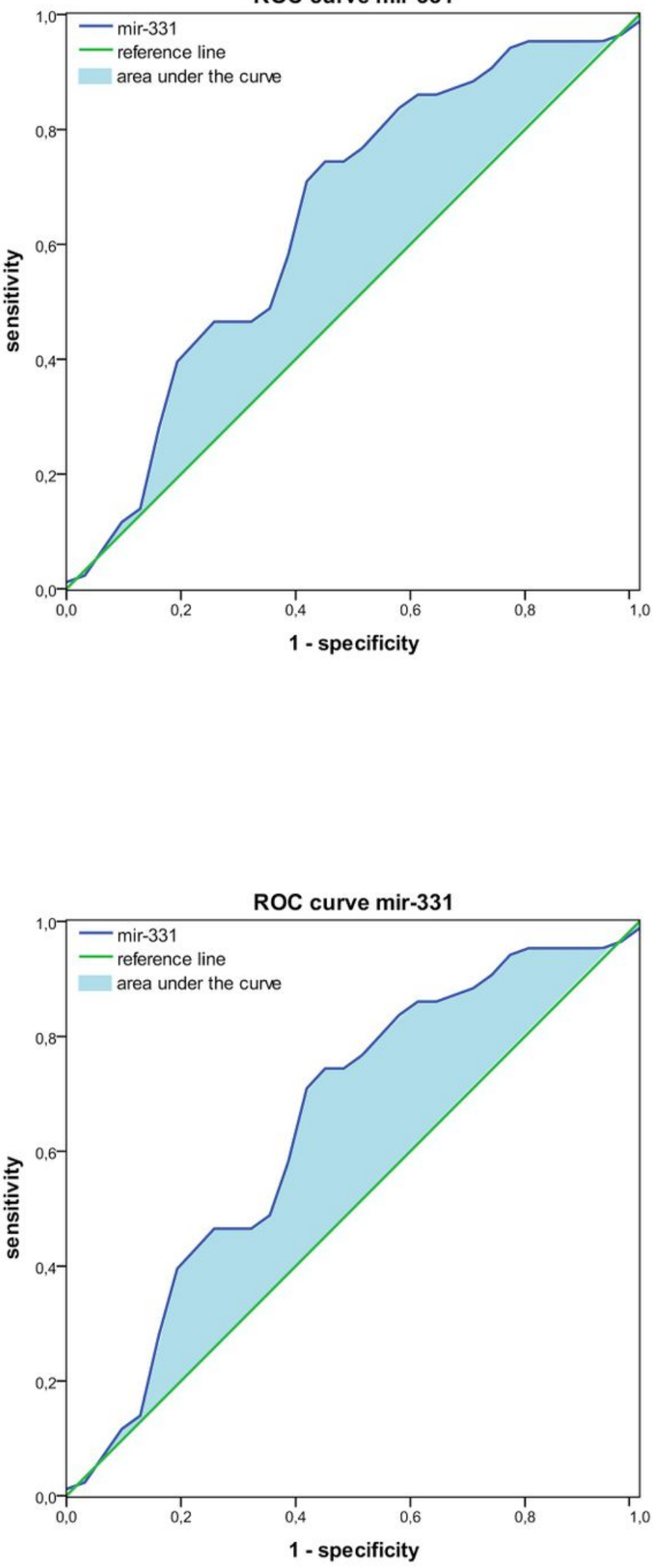

Figure 2 
ROC-curves of hsa-mir-200b and hsa-mir-331

\section{Supplementary Files}

This is a list of supplementary files associated with this preprint. Click to download.

- miRNApapertables20201119.pdf

- miRNApapertables20201119.pdf 